\title{
ON DIRICHLET'S BOUNDARY VALUE PROBLEM FOR SOME FORMALLY HYPOELLIPTIC DIFFERENTIAL OPERATORS
}

\author{
NIELS JACOB \\ (Received 3 March 1986; revised 4 November 1986)
}

Communicated by R. Vyborny

\begin{abstract}
For a class of formally hypoelliptic differential operators in divergence form we prove a generalized Gårding inequality. Using this inequality and further properties of the sesquilinear form generated by the differential operator a generalized homogeneous Dirichlet problem is treated in a suitable Hilbert space. In particular Fredholm's alternative theorem is proved to be valid.
\end{abstract}

1980 Mathematics subject classification (Amer. Math. Soc.): 35 G 15, 35 H 05.

\section{Introduction}

For uniformly strongly elliptic differential operators of order $2 m$ in divergence form, the Dirichlet problem can be treated completely by using Hilbert space methods (see A. Friedman [2], part I, and L. Nirenberg [15]). For these operators one can introduce a suitable Hilbert space, the Sobolev space $H_{0}^{m}(\Omega), \Omega \subset \subset \mathbf{R}^{n}$, compactly imbedded in $L^{2}(\Omega)$ and generalizing homogeneous Dirichlet boundary data. The sesquilinear form generated by the considered operator is continuous in $H_{0}^{m}(\Omega)$ and moreover it satisfies a coercivity inequality, namely Gårding's inequality (see L. Gårding [3]). Using some abstract functional analysis, the two properties of the sesquilinear form mentioned above, and the compactness of the imbedding of $H_{0}^{m}(\Omega)$ in $L^{2}(\Omega)$, one gets an existence result for a generalized Dirichlet problem. Under additional regularity assumptions on the coefficients of

(C) 1988 Australian Mathematical Society $0263-6115 / 88 \$ A 2.00+0.00$ 
the operator, it is then possible to prove interior regularity results of the solutions (Weyl's Lemma) and regularity up to the boundary, provided the boundary is smooth.

There have been several attempts to apply these methods to other boundary value problems for elliptic operators (see M. Schechter [16]--[18] and the references given there) and to other classes of operators. The latter has been done by J. M. Berezanskir for operators with constant coefficients (see [1], Chapter IV), by S. M. Nikol'skii in [14] for a class of symmetric operators with real coefficients, by F. Stummel in [20], Chapter 3.5, for semielliptic operators having coefficients satisfying the same conditions as the coefficients have to fulfill in the elliptic case and for a class of formally selfadjoint hypoelliptic operators with $C^{\infty}$-coefficients by B. Malgrange [12], page 303. For some classes of non-hypoelliptic operators an existence theorem for a generalized Dirichlet problem was given in [10] and [11] under some restricted conditions on the oscillation of the coefficients of a generalized principal part. Later this was also done independently under more restricted conditions on the operators by H.-J. Herrler in [4].

The purpose of this paper is to establish the Hilbert space methods for solving a generalized Dirichlet problem for a large class of operators having divergence structure and the symbol of which are bounded from below and from above by the square of a fixed hypoelliptic polynomial $P(\xi)$ (see C1-C5 below). After we have given some auxiliary results (Section 2 ) we prove a coercivity inequality (Theorems 1 and 2) for the considered operators under the same conditions on the coefficients as one has to pose in the elliptic case (Sections 3 and 4). For this it is necessary to define a generalized principal part and a lower order part of the operator. As the natural space to handle a Dirichlet problem a Hilbert space $H_{0}^{P}(\Omega), \Omega \subset \subset \mathbf{R}^{n}$, appears (see Section 5). Indeed, the sesquilinear form generated by the operator is continuous in $H_{0}^{P}(\Omega)$ (Theorem 5) and the coercivity inequality is nothing but Gårding's inequality. Since the space $H_{0}^{P}(\Omega)$ is compactly imbedded in $L^{2}(\Omega)$ (Theorem 3 ) we can solve the Dirichlet problem in the same way as it is done for elliptic operators (Theorem 6). Moreover it is shown that the space $H_{0}^{P}(\Omega)$ generalizes homogeneous Dirichlet data (Theorem 4). In Section 7 we first show that uniformly strongly elliptic and semielliptic operators in divergence form belong to the class considered here. But we give also an example of a non-semielliptic operator (Example 3). When the operator has continuous coefficients (which is a restriction on the coefficients of the lower order part) we can prove that the operator is of constant strength in $\Omega$ (in the sense of L. Hörmander [8], Definition 13.1.1) and the operator obtained by freezing the coefficients at an arbitrary point $x_{0} \in \Omega$ is hypoelliptic (Proposition 5). Hence, if the coefficients are of class $C^{\infty}$, the operator is hypoelliptic in the sense of L. Hörmander [8], Definition 13.4.3, and interior regularity results for weak solutions can be applied (see [8], Theorem 13.4.1, in this paper stated as Theorem 
7). Since by L. Hörmander ([6], Example 1, page 242) Dirichlet boundary value problems are hypoelliptic, we can also obtain in the case of constant coefficients and plane parts of the boundary regularity up to the boundary. However, we have all estimates needed to carry over the methods used in [2] and [15] to obtain regularity results under much weaker conditions on the coefficients. But this will be done elsewhere.

Finally let us remark that Gårding's inequality can be derived for some hypoelliptic differential operators by methods of the theory of pseudodifferential operators if we suppose the coefficients to be arbitrarily often differentiable. For such inequalities see A. Melin [13] and L. Hörmander [9], Chapter XXII, and the references given there. However it seems that for less regular coefficients (such as we have) these techniques do not apply.

\section{Notations}

We denote by $\mathbf{N}, \mathbf{R}$ and $\mathbf{C}$ the positive integers, the real numbers and the complex numbers, respectively. For $n \in N$ the Euclidean space is denoted by $\mathbf{R}^{n}$. Furthermore let $\mathbf{N}_{0}:=\mathbf{N} \cup\{0\}$ and $\mathbf{N}_{0}^{n}$ be the set of all multi-indices. For $\alpha, \beta \in \mathbf{N}_{0}^{n}$ we set

$$
\begin{aligned}
& \alpha \pm \beta:=\left(\alpha_{1}+\beta_{1}, \ldots, \alpha_{n} \pm \beta_{n}\right) \\
& \alpha \leq \beta \text { if and only if } \alpha_{j} \leq \beta_{j}, \quad 1 \leq j \leq n \\
& |\alpha|:=\alpha_{1}+\cdots+\alpha_{n} \\
& \alpha !:=\alpha_{1} ! \cdots \alpha_{n} ! \\
& \left(\begin{array}{c}
\alpha \\
\beta
\end{array}\right)=\left(\begin{array}{c}
\alpha_{1} \\
\beta_{1}
\end{array}\right) \cdots\left(\begin{array}{c}
\alpha_{n} \\
\beta_{n}
\end{array}\right)
\end{aligned}
$$

and

$$
|\alpha: \beta|:=\sum_{j=1}^{n} \frac{\alpha_{j}}{\beta_{j}} .
$$

The unit vector in the $j$ th direction is denoted by $\varepsilon_{j} \in \mathbf{R}^{n} \cap \mathbf{N}_{0}^{n}$. Moreover for $\xi \in \mathbf{R}^{n}$ and $\alpha \in \mathbf{N}_{0}^{n}$ we define

$$
\xi^{\alpha}:=\xi_{1}^{\alpha_{1}} \cdots \xi_{n}^{\alpha_{n}}
$$

By $\Omega \subset \mathbf{R}^{n}$ we will always denote a bounded open set. For two open sets $\Omega_{1}, \Omega_{2} \subset \mathbf{R}^{n}$ we write $\Omega_{1} \subset \subset \Omega_{2}$ if the closure of $\Omega_{1}$ is compact and contained in $\Omega_{2}$. The space of all functions $\varphi: \Omega \rightarrow \mathbb{C}$ which are arbitrarily often differentiable and have compact support, $\operatorname{supp} \varphi$, in $\Omega$ is denoted by $C_{0}^{\infty}(\Omega)$. The space of 
all $m$-times continuously differentiable functions which, together with all their derivatives of order less or equal to $m$, are bounded is denoted by $\bar{C}^{m}(\Omega)$. The space $L^{2}(\Omega)$ consists of all (equivalence classes of) Lebesgue measurable functions $u: \Omega \rightarrow \mathbb{C}$ having finite norm $\|u\|_{0}^{2}=\int_{\Omega}|u(x)|^{2} d x$. A Lebesgue measurable function belongs to $L^{\infty}(\Omega)$ if and only if $\|u\|_{\infty}=\operatorname{ess} \sup _{x \in \Omega}|u(x)|$ is finite.

Let $P(\xi)$ be a polynomial with complex coefficients. For $\alpha \in \mathbf{N}_{0}^{n}$ we write $P^{(\alpha)}(\xi)$ for the polynomial

$$
\partial_{\xi}^{\alpha} P(\xi)=\frac{\partial^{|\alpha|} P(\xi)}{\partial \xi_{1}^{\alpha_{1}} \cdots \partial \xi_{n}^{\alpha_{n}}}
$$

For $l=1, \ldots, n$ we write $P^{(l)}(\xi)$ for $P^{\left(\varepsilon_{l}\right)}(\xi)$. By $D^{\alpha}$ we denote the differential operator

$$
D^{\alpha}:=\left(-i \frac{\partial}{\partial \xi_{1}}\right)^{\alpha_{1}} \cdots\left(-i \frac{\partial}{\partial \xi_{n}}\right)^{\alpha_{n}}, \quad i^{2}=-1 .
$$

Given a polynomial $P(\xi)=\sum_{\alpha \in \Gamma} a_{\alpha} \xi^{\alpha}, \Gamma \subset \mathbf{N}_{0}^{n}$, we associate with $P(\xi)$ the differential operator $P(D)=\sum_{\alpha \in \Gamma} a_{\alpha} D^{\alpha}$.

Let $\varphi \in C_{0}^{\infty}(\Omega)$. The Fourier transform of $\varphi$ at the point $\xi$ is given by

$$
F \varphi(\xi):=(2 \pi)^{-n / 2} \int_{\Omega} e^{-i x \cdot \xi} \varphi(x) d x .
$$

For a differential operator $P(D)$, we have the important relation

$$
F(P(D) \varphi)(\xi)=P(\xi) F \varphi(\xi) .
$$

Using this formula we can define operators for arbitrary, polynomial bounded functions. On $L^{2}(\Omega)$ a scalar product is given by

$$
(\varphi, \psi)_{0}:=\int_{\Omega} \varphi(x) \overline{\Psi(x)} d x
$$

with corresponding norm $\|\cdot\|_{0}$. When $P(D)$ is a differential operator, Plancherel's theorem gives

$$
\|P(D) \varphi\|_{0}^{2}=\int_{\mathbf{R}^{n}}|P(\xi)|^{2}|F \varphi(\xi)|^{2} d \xi
$$

for each $\varphi \in C_{0}^{\infty}(\Omega)$. We introduce the following family of norms on $C_{0}^{\infty}(\Omega)$. For $s \in \mathbf{R}$ let $\Lambda^{s}(\xi):=\left(1+|\xi|^{2}\right)^{s / 2} ;$ then we define for $\varphi \in C_{0}^{\infty}(\Omega)$

$$
\|\varphi\|_{s}^{2}=\int_{\mathbf{R}^{n}} \Lambda^{2 s}(\xi)|F \varphi(\xi)|^{2} d \xi .
$$

Note, that this is the usual Sobolev-space norm. Finally we mention that $J$ will always denote a finite set of indices. By $\varepsilon$ and $\eta$ we will always mean arbitrary positive numbers. By $c$ we denote often an arbitrary, positive constant which may change from line to line. 


\section{Auxiliary results}

We give some results needed frequently later.

PROPOSITION 1. Let $u$ and $v$ be two arbitrarily often differentiable functions and $P(D)$ a differential operator with constant coefficients. Then Leibniz's rule gives

$$
P(D)(u \cdot v)=\sum_{\alpha \in N_{0}^{n}} \frac{1}{\alpha !} P^{(\alpha)}(D) u D^{\alpha} v .
$$

Note that the above sum is finite. A proof of (2.1) can be found in [7], formula (1.1.10). We often use (2.1) in the form

$$
u P(D) v=P(D)(u \cdot v)-\sum_{\substack{\alpha \in N_{0}^{n} \\ \alpha \neq 0}} \frac{1}{\alpha !} P^{(\alpha)}(D) u D^{\alpha} v
$$

PROPOSITION 2. Let $P(D)$ be a hypoelliptic differential operator, $\Omega \subset \mathbf{R}^{n}$ an open bounded set and $\alpha \in \mathbf{N}_{0}^{n}, \alpha \neq 0$. Then for each $\varepsilon>0$ there exists a constant $c(\varepsilon, \alpha) \geq 0$ such that

$$
\left\|P^{(\alpha)}(D) \varphi\right\|_{0} \leq \varepsilon\|P(D) \varphi\|_{0}+c(\varepsilon, \alpha)\|\varphi\|_{0}
$$

holds for all $\varphi \in C_{0}^{\infty}(\Omega)$.

ProOF. Since $P(D)$ is hypoelliptic, there exists two constants $c_{0}>0$ and $\rho \geq 0$, independently of $\alpha \in \mathbf{N}_{0}^{n}$, such that for all $\xi \in \mathbf{R}^{n},|\xi|>\rho$, the inequality

$$
\frac{\left|P^{(\alpha)}(\xi)\right|}{|P(\xi)|} \leq c_{\alpha}|\xi|^{-|\alpha| c_{0}} \text {. }
$$

holds (see [8], Theorem 11.1.3). Now for $\varphi \in C_{0}^{\infty}(\Omega)$ we have

$$
\begin{aligned}
\left\|P^{(\alpha)}(D) \varphi\right\|_{0}^{2}= & \int_{R^{n}}\left|P^{(\alpha)}(\xi)\right|^{2}|F \varphi(\xi)|^{2} d \xi \\
\leq & \int_{|\xi| \leq \rho}\left|P^{(\alpha)}(\xi)\right|^{2}|F \varphi(\xi)|^{2} d \xi \\
& +c_{\alpha} \int_{|\xi|>\rho}|\xi|^{-2 c_{0}|\alpha|}|P(\xi)|^{2}|F \varphi(\xi)|^{2} d \xi \\
\leq & \tilde{c}_{\alpha}(\rho)\|\varphi\|_{0}^{2}+c_{\alpha} \rho^{-2 c_{0}|\alpha|}\|P(D) \varphi\|_{0}^{2} .
\end{aligned}
$$

Given $\eta>0$, we take $\rho$ such that $c_{\alpha} \rho^{-2 c_{0}|\alpha|} \leq \eta$ holds. Then we get

$$
\left\|P^{(\alpha)}(D) \varphi\right\|_{0}^{2} \leq \eta\|P(D) \varphi\|_{0}^{2}+\tilde{c}(\eta, \alpha)\|\varphi\|_{0}^{2}
$$

which implies (2.3). 
Proposition 3. Let $Q(\xi)$ and $P(\xi)$ be two polynomials and suppose for each $\alpha \in \mathbf{N}_{0}^{n}, \alpha \neq 0$,

$$
\lim _{|\xi| \rightarrow \infty} \frac{\left|Q^{(\alpha)}(\xi)\right|}{|P(\xi)|}=0
$$

to be valid. Then for each $\varepsilon>0$ there exists a constant $c_{\alpha}(\varepsilon)$ such that

$$
\left\|Q^{(\alpha)}(D) \varphi\right\|_{0} \leq \varepsilon\|P(D) \varphi\|_{0}+c_{\alpha}(\varepsilon)\|\varphi\|_{0}
$$

holds for all $\varphi \in C_{0}^{\infty}(\Omega)$.

Proof. From (2.6) we find that for $\eta>0$ there exists a $\rho \geq 0$ such that

$$
\left|Q^{(\alpha)}(\xi)\right|^{2} \leq \eta|P(\xi)|^{2}
$$

holds for all $\xi \in \mathbf{R}^{n},|\xi| \geq \rho$. Therefore we get

$$
\begin{aligned}
\left\|Q^{(\alpha)}(D) \varphi\right\|_{0}^{2} & =\int_{\mathbf{R}^{n}}\left|Q^{(\alpha)}(\xi)\right|^{2}|F \varphi(\xi)|^{2} d \xi \\
& \leq \int_{|\xi| \leq \rho}\left|Q^{(\alpha)}(\xi)\right|^{2}|F \varphi(\xi)|^{2} d \xi+\eta \int_{|\xi|>\rho}|P(\xi)|^{2}|F \varphi(\xi)|^{2} d \xi \\
& \leq \eta\|P(D) \varphi\|_{0}^{2}+\tilde{c}_{\alpha}(\eta)\|\varphi\|_{0}^{2},
\end{aligned}
$$

which gives the proposition.

PROPOSITION 4. Let $\Omega \subset \mathbf{R}^{n}$ be a bounded open set. Suppose in addition that for two polynomials $Q(\xi)$ and $P(\xi)$ the estimate

$$
|Q(\xi)| \leq c|P(\xi)|
$$

holds with some constant $c \geq 0$ and all $\xi \in \mathbf{R}^{n},|\xi| \geq \rho$. Then there is a constant $c^{\prime}$ such that

$$
\|Q(D) \varphi\|_{0} \leq c^{\prime}\|P(D) \varphi\|_{0}
$$

is valid for all $\varphi \in C_{0}^{\infty}(\Omega)$.

ProOF. Notice first that

$$
\begin{aligned}
\|Q(D) \varphi\|_{0}^{2}= & \int_{\mathbf{R}^{n}}|Q(\xi)|^{2}|F \varphi(\xi)|^{2} d \xi \\
\leq & c^{2} \int_{\mathbf{R}^{n}}|P(\xi)|^{2}|F \varphi(\xi)|^{2} d \xi \\
& +\int_{|\xi| \leq \rho}|Q(\xi)|^{2}|F \varphi(\xi)|^{2} d \xi \\
\leq & c^{2}\|P(D) \varphi\|_{0}^{2}+c^{*}\|\varphi\|_{0}^{2} .
\end{aligned}
$$


By Theorem 1.13 in [19] we know also

$$
\|\varphi\|_{0}^{2} \leq \tilde{c}\|P(D) \varphi\|_{0}^{2}
$$

and the proposition is proved.

\section{Gårding's inequality. Part I}

We will prove a generalized Gårding inequality for a differential operator

$$
L_{\mathrm{pr}}(x, D)=\sum_{i, j=1}^{m} P_{i}(D) a_{i j}(x) Q_{j}(D),
$$

where $P_{i}(D)$ and $Q_{j}(D), 1 \leq i, j \leq m$, are differential polynomials with constant real coefficients. The operator $L_{\mathrm{pr}}(x, D)$ will serve as the generalized principal part in the next section. The following conditions are imposed on $L_{\mathrm{pr}}(x, D)$ :

C1. There are two constants $c_{0}>0, \rho_{0} \geq 0$ and a hypoelliptic polynomial $P(\xi)$ such that for all $\xi \in \mathbf{R}^{n},|\xi| \geq \rho_{0}$, the estimate

$$
\operatorname{Re} \sum_{i, j=1}^{m} P_{i}(\xi) a_{i j}(x) Q_{j}(\xi) \geq c_{0}|P(\xi)|^{2}
$$

holds.

C2. Let $P(\xi)$ be the polynomial as in $\mathrm{C} 1$ and let $c^{\prime}$ and $c^{\prime \prime}$ be two non-negative constants. We assume

$$
\left|P_{i}(\xi)\right| \leq c^{\prime}|P(\xi)|, \quad i=1, \ldots, m,
$$

and

$$
\left|Q_{j}(\xi)\right| \leq c^{\prime \prime}|P(\xi)|, \quad j=1, \ldots, m,
$$

for all $\xi \in \mathbf{R}^{n}$ with $|\xi|$ sufficiently large.

C3. For $\alpha \in \mathbf{N}_{0}^{n}, \alpha \neq 0$, and $1 \leq i, j \leq m$

$$
\lim _{|\xi| \rightarrow \infty} \frac{\left|P_{i}^{(\alpha)}(\xi)\right|}{|P(\xi)|}=\lim _{|\xi| \rightarrow \infty} \frac{\left|Q_{j}^{(\alpha)}(\xi)\right|}{|P(\xi)|}=0
$$

is required.

C4. The functions $a_{i j}, 1 \leq i, j \leq m$, are defined on an open bounded set $\Omega$ and take values in $C$. Furthermore we assume

$$
\sup _{x \in \Omega}\left|a_{i j}(x)\right| \leq M, \quad 1 \leq i, j \leq m,
$$


and

$$
\left|a_{i j}(x)-a_{i j}(y)\right| \leq f(|x-y|), \quad 1 \leq i, j \leq m,
$$

for all $x, y \in \Omega$. Here $f: \mathbf{R}^{+} \rightarrow \mathbf{R}$ is a function satisfying $\lim _{t \rightarrow 0} f(t)=0$.

THEOREM 1. Suppose that for the differential operator (3.1) the conditions $\mathrm{C} 1-\mathrm{C} 4$ are fulfilled. Then for all $\varphi \in C_{0}^{\infty}(\Omega)$ we have

$$
\begin{aligned}
& \operatorname{Re} \sum_{i, j=1}^{m} \int_{\Omega} a_{i j}(x) Q_{j}(D) \varphi(x) \overline{P_{i}(D) \varphi(x)} d x \\
& \quad \geq\left(c_{0} / 4\right)\|P(D) \varphi\|_{0}^{2}-\tilde{c}\|\varphi\|_{0}^{2},
\end{aligned}
$$

where $c_{0}$ is the constant in (3.2) and $\tilde{c} \geq 0$ is another constant.

ProOF. We prove (3.8) in three steps.

1. In this step we assume the coefficients $a_{i j}, 1 \leq i, j \leq m$, to be complex constants. Then Plancherel's theorem gives

$$
\begin{aligned}
& \operatorname{Re} \sum_{i, j=1}^{m} \int_{\Omega} a_{i j} Q_{j}(D) \varphi(x) \overline{P_{i}(D) \varphi(x)} d x \\
& \quad=\int_{\mathbf{R}^{n}} \operatorname{Re} \sum_{i, j=1}^{m} a_{i j} Q_{j}(\xi) P_{i}(\xi)|F \varphi(\xi)|^{2} d \xi
\end{aligned}
$$

By $\mathrm{C} 1$ and (3.6) we get immediately

$$
\begin{aligned}
\operatorname{Re} & \sum_{i, j=1}^{m} \int_{\Omega} a_{i j} Q_{j}(D) \varphi(x) \overline{P_{i}(D) \varphi(x)} d x \\
& \geq c_{0} \int_{|\xi| \geq \rho_{0}}|P(\xi)|^{2}|F \varphi(\xi)|^{2} d \xi-c \int_{|\xi|<\rho_{0}}|F \varphi(\xi)|^{2} d \xi \\
& \geq c_{0}\|P(D) \varphi\|_{0}^{2}-\tilde{c}_{1}\|\varphi\|_{0}^{2} .
\end{aligned}
$$

2. Now, let $x_{0} \in \Omega$ be a fixed point and $U \subset \Omega$ an open neighbourhood of $x_{0}$ such that

$$
K^{2} m^{2} \max _{1 \leq i, j \leq m} \max _{x, y \in U}\left|a_{i j}(x)-a_{i j}(y)\right| \leq c_{0} / 2
$$

holds. By $K$ we denote the greatest possible constant in (2.10) which we get by applying Proposition 4 to the operators $Q_{j}(D)$ and $P_{i}(D), 1 \leq i, j \leq m$. We will prove (3.8) for those $\varphi \in C_{0}^{\infty}(\Omega)$ with $\operatorname{supp} \varphi \subset U$. By the result of the first 
step we get

$$
\begin{aligned}
\operatorname{Re} \sum_{i, j=1}^{m} \int_{\Omega} a_{i j}(x) Q_{j}(D) \varphi(x) \overline{P_{i}(D) \varphi(x)} d x \\
=\operatorname{Re} \sum_{i, j=1}^{m} \int_{\Omega} a_{i j}\left(x_{0}\right) Q_{j}(D) \varphi(x) \overline{P_{i}(D) \varphi(x)} d x \\
\quad+\operatorname{Re} \sum_{i, j=1}^{m} \int_{\Omega}\left(a_{i j}(x)-a_{i j}\left(x_{0}\right)\right) Q_{j}(D) \varphi(x) \overline{P_{i}(D) \varphi(x)} d x \\
\geq c_{0}\|P(D) \varphi\|_{0}^{2}-\tilde{c}_{1}\|\varphi\|_{0}^{2} \\
\quad-\sum_{i, j=1}^{m} \int_{\Omega}\left|a_{i j}(x)-a_{i j}\left(x_{0}\right)\left\|Q_{j}(D) \varphi(x)\right\| P_{i}(D) \varphi(x)\right| d x \\
\geq c_{0}\|P(D) \varphi\|_{0}^{2}-\tilde{c}\|\varphi\|_{0}^{2} \\
\quad-\max _{1 \leq i, j \leq m} \max _{x, y \in U}\left|a_{i j}(x)-a_{i j}(y)\right| \sum_{i, j=1}^{m} \int_{\Omega}\left|Q_{j}(D) \varphi(x) \| P_{i}(D) \varphi(x)\right| d x \\
\geq c_{0}\|P(D) \varphi\|_{0}^{2}-\tilde{c}_{1}\|\varphi\|_{0}^{2}-\frac{c_{0}}{2 K^{2} m^{2}} \sum_{i, j=1}^{m}\left\|Q_{j}(D) \varphi\right\|_{0}\left\|P_{i}(D) \varphi\right\|_{0},
\end{aligned}
$$

where we used for the last estimate (3.9) and the Cauchy-Schwarz inequality. Using condition C2 we can apply Proposition 4 to estimate $\left\|Q_{j}(D) \varphi\right\|_{0}, 1 \leq j \leq$ $m$, and $\left\|P_{i}(D) \varphi\right\|_{0}, 1 \leq i \leq m$. With $K$ defined as in (3.9) we get

$$
\left\|Q_{j}(D) \varphi\right\|_{0}\left\|P_{i}(D) \varphi\right\|_{0} \leq K^{2}\|P(D) \varphi\|_{0}^{2}
$$

for $1 \leq i, j \leq m$. Finally, it follows that

$$
\begin{aligned}
\operatorname{Re} & \sum_{i, j=1}^{m} \int_{\Omega} a_{i j}(x) Q_{j}(D) \varphi(x) \overline{P_{i}(D) \varphi(x)} d x \\
\geq & c_{0}\|P(D) \varphi\|_{0}^{2}-\tilde{c}_{1}\|\varphi\|_{0}^{2} \\
& \quad-\frac{c_{0}}{2 K^{2} m^{2}} \sum_{i, j=1}^{m} K^{2}\|P(D) \varphi\|_{0}^{2} \\
& =\frac{c_{0}}{2}\|P(D) \varphi\|_{0}^{2}-\tilde{c}_{2}\|\varphi\|_{0}^{2},
\end{aligned}
$$

hence the second step is finished.

3. Let $\left(U_{k}\right)_{k \in J}$ be a finite covering of $\bar{\Omega}$ with open sets, such that for each $k \in J$ we have

$$
K^{2} m^{2} \max _{1 \leq i, j \leq m} \max _{x, y \in U_{k}}\left|a_{i j}(x)-a_{i j}(y)\right| \leq c_{0} / 2 .
$$

Again $K$ is the same constant as it is in (3.9). Furthermore let $\left(b_{k}\right)_{k \in J}$ be a partition of unity subordinate to the covering $\left(U_{k}\right)_{k \in J}$. In addition we assume 
that for each $k \in J$ the function $a_{k}=\sqrt{b_{k}}$ is arbitrarily often differentiable. ${ }^{1}$ Now, we get

$$
\begin{aligned}
\operatorname{Re} & \sum_{i, j=1}^{m} \int_{\Omega} a_{i j}(x) Q_{j}(D) \varphi(x) \overline{P_{i}(D) \varphi(x)} d x \\
\quad= & \operatorname{Re} \sum_{i, j=1}^{m} \sum_{k \in J} \int_{\Omega} a_{k}^{2}(x) a_{i j}(x) Q_{j}(D) \varphi(x) \overline{P_{i}(D) \varphi(x)} d x
\end{aligned}
$$

With formula (2.2) we obtain

$$
a_{k}(x) Q_{j}(D) \varphi(x)=Q_{j}(D)\left(a_{k}(x) \varphi(x)\right)-\sum_{\substack{\sigma \in \mathbb{N}^{n} \\ \sigma \neq 0}} \frac{1}{\sigma !}\left(Q_{j}^{(\sigma)}(D) \varphi(x)\right) D^{\sigma} a_{k}(x)
$$

and

$$
a_{k}(x) P_{i}(D) \varphi(x)=P_{i}(D)\left(a_{k}(x) \varphi(x)\right)-\sum_{\substack{\tau \in N_{0}^{n} \\ \tau \neq 0}} \frac{1}{\tau !}\left(P_{i}^{(\tau)}(D) \varphi(x)\right) D^{\tau} a_{k}(x) .
$$

From this it follows that

$$
\begin{aligned}
& \operatorname{Re} \sum_{i, j=1}^{m} \int_{\Omega} a_{i j}(x) Q_{j}(D) \varphi(x) \overline{P_{i}(D) \varphi(x)} d x \\
& \quad=A_{1}+A_{2}+A_{3}+A_{4} \geq A_{1}-\left|A_{2}\right|-\left|A_{3}\right|-\left|A_{4}\right|,
\end{aligned}
$$

where

$$
\begin{aligned}
& A_{1}=\operatorname{Re} \sum_{i, j=1}^{m} \sum_{k \in J} \int_{\Omega} a_{i j}(x) Q_{j}(D)\left(a_{k}(x) \varphi(x)\right) \overline{P_{i}(D)\left(a_{k}(x) \varphi(x)\right)} d x \\
& A_{2}=-\operatorname{Re} \sum_{i, j=1}^{m} \sum_{k \in J} \sum_{\substack{\sigma \in \mathbb{N}_{0}^{n} \\
\sigma \neq 0}} \int_{\Omega} \frac{1}{\sigma !} a_{i j}(x)\left(Q_{j}^{(\sigma)}(D) \varphi(x)\right)\left(D^{\sigma} a_{k}(x)\right) \overline{P_{i}(D) \varphi(x)} d x \\
& A_{3}=-\operatorname{Re} \sum_{i, j=1}^{m} \sum_{k \in J} \sum_{\substack{\tau \in N_{0}^{n} \\
\tau \neq 0}} \int_{\Omega} \frac{1}{\tau !} a_{i j}(x) Q_{j}(D) \varphi(x)\left(P_{i}^{(\tau)}(D) \varphi(x)\right) D^{\tau} a_{k}(x) d x
\end{aligned}
$$

and

$$
\begin{aligned}
A_{4}=\operatorname{Re} \sum_{i, j=1}^{m} \sum_{k \in J} \sum_{\substack{\sigma, \tau \in \mathcal{N}^{n} \\
\sigma \neq 0, \tau \neq 0}} \int_{\Omega} \frac{1}{\sigma ! \tau !} a_{i j}(x)\left(Q_{j}^{(\sigma)}(D) \varphi(x)\right) \\
\quad \times\left(D^{\sigma} a_{k}(x)\right) \overline{\left(P_{i}^{(\tau)}(D) \varphi(x)\right)} \overline{D^{\tau} a_{k}(x)} d x .
\end{aligned}
$$

${ }^{1}$ Following [2], p. 35, it is always possible to assume $\sqrt{b}_{k} \in C^{\infty}$. This can be proved by replacing $b_{k}$ by $b_{k}^{2} /\left(\sum_{k \in J} b_{k}^{2}\right)$. 
By our second step, we find

$$
\begin{aligned}
A_{1} & \geq \sum_{k \in J}\left(\left(c_{0} / 2\right)\left\|P(D)\left(a_{k} \varphi\right)\right\|_{0}^{2}-\tilde{c}_{2}\left\|a_{k} \varphi\right\|_{0}^{2}\right) \\
& \geq\left(c_{0} / 2\right) \sum_{k \in J}\left\|P(D)\left(a_{k} \varphi\right)\right\|_{0}^{2}-\hat{c}_{2}\|\varphi\|_{0}^{2}
\end{aligned}
$$

We estimate $A_{2}$. First we get

$$
\begin{aligned}
\left|A_{2}\right| & \leq c_{1} \sum_{i, j=1}^{m} \sum_{\substack{\sigma \in \mathbb{N}_{0}^{n} \\
\sigma \neq 0}} \int_{\Omega}\left|Q_{j}^{(\sigma)}(D) \varphi(x) \| P_{i}(D) \varphi(x)\right| d x \\
& \leq c_{1} \sum_{i, j=1}^{m} \sum_{\substack{\sigma \in N_{0}^{n} \\
\sigma \neq 0}}\left\|Q_{j}^{(\sigma)}(D) \varphi\right\|_{0}\left\|P_{i}(D) \varphi\right\|_{0} .
\end{aligned}
$$

By Propositions 3 and 4, and by C2 and C3 for each $\eta>0$ it follows that

$$
\left|A_{2}\right| \leq c_{2} \sum_{i, j=1}^{m}\left(\eta\|P(D) \varphi\|_{0}+c^{j}(\eta)\|\varphi\|_{0}\right)\|P(D) \varphi\|_{0}
$$

Since for $\eta>0$ we have $|a||b| \leq \eta|a|^{2}+1 / 4 \eta|b|^{2}$ we can find for each $\varepsilon>0$ a number $\eta>0$ such that

$$
\left|A_{2}\right| \leq \varepsilon\|P(D) \varphi\|_{0}^{2}+c_{2}^{\prime}(\varepsilon)\|\varphi\|_{0}^{2}
$$

holds. Similarly we can handle $A_{3}$ to get for all $\varepsilon>0$ the inequality

$$
\left|A_{3}\right| \leq \varepsilon\|P(D) \varphi\|_{0}^{2}+c_{3}^{\prime}(\varepsilon)\|\varphi\|_{0}^{2} .
$$

In order to estimate $A_{4}$, notice that

$$
\begin{aligned}
\left|A_{4}\right| & \leq c_{5} \sum_{i, j=1}^{m} \sum_{\substack{\sigma, \tau \in N_{0}^{n} \\
\sigma \neq 0, \tau \neq 0}} \int_{\Omega}\left|Q_{j}^{(\sigma)}(D) \varphi(x) \| P_{i}^{(\tau)}(D) \varphi(x)\right| d x \\
& \leq c_{5} \sum_{i, j=1}^{m} \sum_{\substack{\sigma, \tau \in N_{0}^{n} \\
\sigma \neq 0, \tau \neq 0}}\left\|Q_{j}^{(\sigma)}(D) \varphi\right\|_{0}\left\|P_{i}^{(\tau)}(D) \varphi\right\|_{0} .
\end{aligned}
$$

Using Proposition 3 and C3 we get for $\eta>0$

$$
\left|A_{4}\right| \leq c_{6} \sum_{i, j=1}^{m}\left(\eta\|P(D) \varphi\|_{0}+\tilde{c}^{j}(\eta)\|\varphi\|_{0}\right)\left(\eta\|P(D) \varphi\|_{0}+\tilde{c}^{i}(\eta)\|\varphi\|_{0}\right) .
$$

Hence, for $\varepsilon>0$ we find

$$
\left|A_{4}\right| \leq \varepsilon\|P(D) \varphi\|_{0}^{2}+c_{4}^{\prime}(\varepsilon)\|\varphi\|_{0}^{2} .
$$


So far we have proved for each $\varepsilon>0$ the estimate

$$
\begin{aligned}
\operatorname{Re} & \sum_{i, j=1}^{m} \int_{\Omega} a_{i j}(x) Q_{j}(D) \varphi(x) \overline{P_{i}(D) \varphi(x)} d x \\
\geq & \left(c_{0} / 2\right) \sum_{k \in J}\left\|P(D)\left(a_{k} \varphi\right)\right\|_{0}^{2}-3 \varepsilon\|P(D) \varphi\|_{0}^{2} \\
& \quad-\left(\hat{c}_{2}+c_{2}^{\prime}(\varepsilon)+c_{3}^{\prime}(\varepsilon)+c_{4}^{\prime}(\varepsilon)\right)\|\varphi\|_{0}^{2} .
\end{aligned}
$$

Let us consider the term

$$
\sum_{k \in J}\left\|P(D)\left(a_{k} \varphi\right)\right\|_{0}^{2}
$$

By Leibniz's rule we get

$$
P(D)\left(a_{k}(x) \varphi(x)\right)=a_{k}(x) P(D) \varphi(x)+\sum_{\substack{\sigma \in N_{0}^{n} \\ \sigma \neq 0}} \frac{1}{\sigma !}\left(P^{(\sigma)}(D) \varphi(x)\right)\left(D^{\sigma} a_{k}(x)\right),
$$

and when we take the sum over $k, k \in J$, it follows that

$$
\sum_{k \in J}\left\|P(D)\left(a_{k} \varphi\right)\right\|_{0}^{2}=B_{1}+B_{2}+B_{3}+B_{4}
$$

where

$$
\begin{aligned}
& B_{1}=\sum_{k \in J} \int_{\Omega} a_{k}^{2}(x)|P(D) \varphi(x)|^{2} d x=\|P(D) \varphi\|_{0}^{2} \\
& B_{2}=\sum_{k \in J} \sum_{\substack{\sigma \in N_{0}^{n} \\
\sigma \neq 0}} \int_{\Omega} \frac{1}{\sigma !} a_{k}(x)(P(D) \varphi(x)) \overline{\left(D^{\sigma} a_{k}(x)\right)} \overline{P^{(\sigma)}(D) \varphi(x)} d x \\
& B_{3}=\sum_{k \in J} \sum_{\substack{\sigma \in \mathbb{N}_{0}^{n} \\
\sigma \neq 0}} \int_{\Omega} \frac{1}{\sigma !} a_{k}(x)\left(D^{\sigma} a_{k}(x)\right)\left(P^{(\sigma)}(D) \varphi(x)\right) \overline{P(D) \varphi(x)} d x
\end{aligned}
$$

and

$$
B_{4}=\sum_{k \in J} \sum_{\substack{\sigma, \tau \in N_{0}^{n} \\ \sigma \neq 0, \tau \neq 0}} \int_{\Omega} \frac{1}{\sigma ! \tau !}\left(D^{\sigma} a_{k}(x)\right)\left(D^{\tau} a_{k}(x)\right)\left(P^{(\sigma)}(D) \varphi(x)\right) \overline{P^{(\tau)}(D) \varphi(x)} d x .
$$

For $B_{2}$ we find

$$
\left|B_{2}\right| \leq c_{7} \sum_{\sigma \in N_{0}^{n}}\|P(D) \varphi\|_{0}\left\|P^{(\sigma)}(D) \varphi\right\|_{0} .
$$

The hypoellipticity of $P(D)$ implies by Proposition 2 for each $\eta>0$

$$
\left|B_{2}\right| \leq c_{7} \sum_{\substack{\sigma \in N_{0}^{n} \\ \sigma \neq 0}}\|P(D) \varphi\|_{0}\left(\eta\|P(D) \varphi\|_{0}+c^{\sigma}(\eta)\|\varphi\|_{0}\right),
$$


which gives for each $\varepsilon>0$

$$
\left|B_{2}\right| \leq \varepsilon\|P(D) \varphi\|_{0}^{2}+c_{5}^{\prime}(\varepsilon)\|\varphi\|_{0}^{2} .
$$

Since also the estimate

$$
\left|B_{3}\right| \leq c_{8} \sum_{\substack{\sigma \in N_{0}^{n} \\ \sigma \in 0}}\left\|P^{(\sigma)}(D) \varphi\right\|_{0}\|P(D) \varphi\|_{0}
$$

holds, we get as before for each $\varepsilon>0$

$$
\left|B_{3}\right| \leq \varepsilon\|P(D) \varphi\|_{0}^{2}+c_{6}^{\prime}(\varepsilon)\|\varphi\|_{0}^{2} .
$$

By Proposition 2 and the obvious estimate

$$
\left|B_{4}\right| \leq c_{9} \sum_{\substack{\sigma, \tau \in N_{0}^{n} \\ \sigma \neq 0, \tau \neq 0}}\left\|P^{(\sigma)}(D) \varphi\right\|_{0}\left\|P^{(\tau)}(D) \varphi\right\|_{0}
$$

we get finally for each $\varepsilon>0$

$$
\left|B_{4}\right| \leq \varepsilon\|P(D) \varphi\|_{0}^{2}+c_{7}^{\prime}(\varepsilon)\|\varphi\|_{0}^{2} .
$$

Now, given $\varepsilon>0$, then it follows that

$$
\begin{aligned}
\operatorname{Re} \sum_{i, j=1}^{m} & \int_{\Omega} a_{i j}(x) Q_{j}(D) \varphi(x) \overline{P_{i}(D) \varphi(x)} d x \\
\geq & \left(c_{0} / 2\right)\left(B_{1}-\left|B_{2}\right|-\left|B_{3}\right|-\left|B_{4}\right|\right) \\
& \quad-3 \varepsilon\|P(D) \varphi\|_{0}^{2}-\left(\hat{c}_{2}+c_{2}^{\prime}(\varepsilon)+c_{3}^{\prime}(\varepsilon)+c_{4}^{\prime}(\varepsilon)\right)\|\varphi\|_{0}^{2} \\
& \geq\left(c_{0} / 2-\left(3 c_{0} / 2+3\right) \varepsilon\right)\|P(D) \varphi\|_{0}^{2}-\left(\hat{c}_{2}+\sum_{l=2}^{7} c_{l}^{\prime}(\varepsilon)\right)\|\varphi\|_{0}^{2} .
\end{aligned}
$$

For $\varepsilon=\left(6 c_{0}+12\right) / c_{0}$ the desired inequality follows with $c=\tilde{c}_{2}+\sum_{l=2}^{7} c_{l}^{\prime}$.

\section{Appendix to Section 3}

We want to prove Theorem 1 for a class of differential operators (3.1) satisfying C2-C4 but instead of $\mathrm{C} 1$ the condition

$\mathrm{C1}^{\prime}$. There are constants $c_{0}>0, \rho_{0} \geq 0$ and a function $P: \mathbf{R}^{n} \rightarrow \mathbb{C}, P \in$ $C^{\infty}\left(\mathbf{R}^{n}\right)$ such that for all $\xi \in \mathbf{R}^{n},|\xi| \geq \rho_{0}$, the estimate (3.2) holds. For the function $P$ there exists a finite set $\Gamma \subset \mathrm{N}_{0}^{n}$, such that the norms $\|P(D) \varphi\|_{0}^{2}$ and $\sum_{\alpha \in \Gamma}\left\|D^{\alpha} \varphi\right\|_{0}^{2}$ are equivalent on $C_{0}^{\infty}(\Omega)$. In addition for each $\alpha \in \Gamma$ and all $\beta \subset N_{0}^{n}, \beta \neq 0$ and $\alpha-\beta \in N_{0}^{n}$, the estimate

$$
\left\|D^{\alpha-\beta} \varphi\right\|_{0} \leq \varepsilon\|P(D) \varphi\|_{0}+c(\varepsilon)\|\varphi\|_{0}
$$

is assumed to hold for all $\varepsilon>0$. 
THEOREM $1^{\prime}$. Suppose that for the differential operator (3.1) the conditions $\mathrm{C1}^{\prime}, \mathrm{C} 2-\mathrm{C} 4$ are fulfilled. Then for all $\varphi \in C_{0}^{\infty}(\Omega)$ inequality $(3.8)$ holds.

ProOF. The proof of Theorem 1 is still valid, but since now the sum

$$
\sum_{\substack{\sigma \in \mathbb{N}_{0}^{n} \\ \sigma \neq 0}} \frac{1}{\sigma !} P^{(\sigma)}(D) \varphi(x) D^{\sigma} a_{k}(x)
$$

is not finite, we have to handle the sum $\sum_{k \in J}\left\|P(D)\left(a_{k} \varphi\right)\right\|_{0}^{2}$ in a modified way. By our assumptions there exists a constant $c^{+}$such that

$$
\sum_{k \in J}\left\|P(D)\left(a_{k} \varphi\right)\right\|_{0}^{2} \geq c^{+} \sum_{k \in J} \sum_{a \in \Gamma}\left\|D^{\alpha}\left(a_{k} \varphi\right)\right\|_{0}^{2} .
$$

Furthermore, for $\alpha \in \Gamma$ we find

$$
\begin{aligned}
\left\|D^{\alpha}\left(a_{k} \varphi\right)\right\|_{0}^{2} & =\int_{\Omega}\left(\sum_{\sigma \leq \alpha}\left(\begin{array}{l}
\alpha \\
\sigma
\end{array}\right) D^{\alpha-\sigma} \varphi(x) D^{\sigma} a_{k}(x)\right) \\
& \times\left(\sum_{\tau \leq \alpha}\left(\begin{array}{l}
\alpha \\
\tau
\end{array}\right) \overline{D^{\alpha-\tau} \varphi(x) D^{\tau} a_{k}(x)}\right) d x \\
& =\int_{\Omega} a_{k}^{2}(x)\left|D^{\alpha} \varphi(x)\right|^{2} d x+C_{2}^{\alpha, k}+C_{3}^{\alpha, k}+C_{4}^{\alpha, k}
\end{aligned}
$$

where

$$
\begin{aligned}
C_{2}^{\alpha, k} & =\int_{\Omega}\left(\sum_{\substack{\sigma \leq \alpha \\
\sigma \neq 0}}\left(\begin{array}{c}
\alpha \\
\sigma
\end{array}\right) D^{\alpha-\sigma} \varphi(x) D^{\sigma} a_{k}(x)\right) a_{k}(x) \overline{D^{\alpha} \varphi(x)} d x, \\
C_{3}^{\alpha, k} & =\int_{\Omega} a_{k}(x) D^{\alpha} \varphi(x)\left(\sum_{\substack{\tau \leq \alpha \\
\tau \neq 0}}\left(\begin{array}{c}
\alpha \\
\tau
\end{array}\right) \overline{D^{\alpha-\tau} \varphi(x) D^{\tau} a_{k}(x)}\right) d x
\end{aligned}
$$

and

$$
\begin{aligned}
& C_{4}^{\alpha, k}=\int_{\Omega}\left(\sum_{\substack{\sigma \leq \alpha \\
\sigma \neq 0}}\left(\begin{array}{l}
\alpha \\
\sigma
\end{array}\right) D^{\alpha-\sigma} \varphi(x) D^{\sigma} a_{k}(x)\right) \\
& \times\left(\sum_{\substack{\tau \leq \alpha \\
\tau \neq \sigma}}\left(\begin{array}{l}
\alpha \\
\tau
\end{array}\right) \overline{D^{\alpha-\tau} \varphi(x) D^{\tau} a_{k}(x)}\right) d x .
\end{aligned}
$$


Using $\mathrm{C} 1^{\prime}$ we estimate $C_{2}^{\alpha, k}$

$$
\begin{aligned}
\left|C_{2}^{\alpha, k}\right| & \leq d_{1} \sum_{\substack{\sigma \leq \alpha \\
\sigma \neq 0}}\left\|D^{\alpha-\sigma} \varphi\right\|_{0}\left\|D^{\alpha} \varphi\right\|_{0} \\
& \leq d_{1} \sum_{\substack{\sigma \leq \alpha \\
\sigma \neq 0}}\left(\eta\|P(D) \varphi\|_{0}+c^{\sigma}(\eta)\|\varphi\|_{0}\right)\left\|D^{\alpha} \varphi\right\|_{0} \\
& \leq d_{2} \sum_{\substack{\sigma \leq \alpha \\
\sigma \neq 0}}\left(\eta\|P(D) \varphi\|_{0}+c^{\sigma}(\eta)\|\varphi\|_{0}\right)\|P(D) \varphi\|_{0},
\end{aligned}
$$

where $\eta>0$ is an arbitrary number. Hence, we get for each $\varepsilon>0$

$$
\left|C_{2}^{\alpha, k}\right| \leq \varepsilon\|P(D) \varphi\|_{0}^{2}+\hat{d}_{2}(\varepsilon)\|\varphi\|_{0}^{2}
$$

and analogously

$$
\left|C_{3}^{\alpha, k}\right| \leq \varepsilon\|P(D) \varphi\|_{0}^{2}+\hat{d}_{3}(\varepsilon)\|\varphi\|_{0}^{2}
$$

Finally it follows that

$$
\left|C_{4}^{\alpha, k}\right| \leq d_{4}\left(\sum_{\substack{\sigma \leq \alpha \\ \sigma \neq 0}}\left\|D^{\alpha-\sigma} \varphi\right\|_{0}\right)\left(\sum_{\substack{\tau \leq \alpha \\ \tau \neq 0}}\left\|D^{\alpha-\tau} \varphi\right\|_{0}\right)
$$

and again using $\mathrm{C1}^{\prime}$ we find for each $\eta>0$

$$
\left|C_{4}^{\alpha, k}\right| \leq d_{4}\left(\sum_{\substack{\sigma \leq \alpha \\ \sigma \neq 0}} \eta\|P(D) \varphi\|_{0}+c^{\alpha}(\eta)\|\varphi\|_{0}\right)\left(\sum_{\substack{\tau \leq \alpha \\ \tau \neq 0}} \eta\|P(D) \varphi\|_{0}+c^{\tau}(\eta)\|\varphi\|_{0}\right)
$$

which gives, for each $\varepsilon>0$, the estimate

$$
\left|C_{4}^{\alpha, k}\right| \leq \varepsilon\|P(D) \varphi\|_{0}^{2}+\hat{d}_{4}(\varepsilon)\|\varphi\|_{0}^{2} .
$$

Now we have

$$
\begin{aligned}
& \sum_{k \in J}\left\|P(D)\left(a_{k} \varphi\right)\right\|_{0}^{2} \geq c^{+} \sum_{k \in J} \sum_{\alpha \in \Gamma}\left\|D^{\alpha}\left(a_{k} \varphi\right)\right\|_{0}^{2} \\
& \geq c^{+} \sum_{k \in J} \sum_{\alpha \in \Gamma} \int_{\Omega} a_{k}^{2}(x)\left|D^{\alpha} \varphi(x)\right|^{2} d x \\
&-c^{+} \sum_{k \in J} \sum_{\alpha \in \Gamma}\left(\left|C_{2}^{\alpha, k}\right|+\left|C_{3}^{\alpha, k}\right|+\left|C_{4}^{\alpha, k}\right|\right) \\
& \geq c^{+} \sum_{\alpha \in \Gamma}\left\|D^{\alpha} \varphi\right\|_{0}^{2}-R \varepsilon\|P(D) \varphi\|_{0}^{2}-\tilde{R}(\varepsilon)\|\varphi\|_{0}^{2}
\end{aligned}
$$

where $R=3|J||\Gamma|$ and $\tilde{R}(\varepsilon)$ is a suitable constant. By $\mathrm{C1}^{\prime}$ we have

$$
\sum_{\alpha \in \Gamma}\left\|D^{\alpha} \varphi\right\|_{0}^{2} \geq c^{-}\|P(D) \varphi\|_{0}^{2}
$$


and by choosing $\varepsilon>0$ appropriately we get

$$
\sum_{k \in J}\left\|P(D)\left(a_{k} \varphi\right)\right\|_{0}^{2} \geq\left(c_{0} / 2\right)\|P(D) \varphi\|_{0}^{2}-\tilde{d}\|\varphi\|_{0}^{2} .
$$

Now we can again follow the proof of Theorem 1 to get Theorem 1'.

\section{Gårding's inequality. Part II}

Let $L(x, D)$ be a differential operator of the form

$$
L(x, D)=L_{\mathrm{pr}}(x, D)+L_{r}(x, D)
$$

where $L_{\mathrm{pr}}(x, D)$ is a differential operator of form (3.1) satisfying C1-C4. The operator $L_{r}(x, D)$ is given by

$$
L_{r}(x, D)=\sum_{\alpha, \beta \in \Gamma^{\prime}} D^{\alpha} a_{\alpha \beta}(x) D^{\beta},
$$

where $\Gamma^{\prime}$ is a finite subset of $\mathrm{N}_{0}^{n}$. The coefficients $a_{\alpha \beta}, \alpha, \beta \in \Gamma^{\prime}$, are supposed to belong to $L^{\infty}(\Omega)$. Furthermore we assume

C5. The operator $L_{r}(x, D)$ can be written as

$$
L_{r}(x, D)=\sum_{\substack{\alpha \in \Gamma_{11} \\ \beta \in \Gamma_{12}}} D^{\alpha} a_{\alpha \beta}(x) D^{\beta}+\sum_{\substack{\alpha \in \Gamma_{21} \\ \beta \in \Gamma_{22}}} D^{\alpha} a_{\alpha \beta}(x) D^{\beta}+\sum_{\alpha, \beta \in \Gamma_{3}} D^{\alpha} a_{\alpha \beta}(x) D^{\beta}
$$

and with the polynomial $P(\xi)$ from $\mathrm{C} 1$ we require for $\alpha \in \Gamma_{11} \cup \Gamma_{22}$

$$
\xi^{2 \alpha} \leq c|P(\xi)|^{2}
$$

for all $\xi \in \mathbf{R}^{n},|\xi|$ sufficiently large, and for $\alpha \in \Gamma_{12} \cup \Gamma_{21} \cup \Gamma_{3}$

$$
\xi^{2 \alpha} \leq c \sum_{l=1}^{n}\left|P^{(l)}(\xi)\right|^{2}
$$

for all $\xi \in \mathbf{R}^{n},|\xi|$ sufficiently large. We have

THEOREM 2. Let $L(x, D)$ be given by (4.1) and suppose C1-C5 to be valid. Then for all $\varphi \in C_{0}^{\infty}(\Omega), \Omega \subset \subset \mathbf{R}^{n}$, we have

$$
\operatorname{Re}(L(x, D) \varphi, \varphi)_{0} \geq\left(c_{0} / 8\right)\|P(D) \varphi\|_{0}^{2}-\tilde{c}_{1}\|\varphi\|_{0}^{2} .
$$

Here $c_{0}$ is the constant in (3.2) and $\tilde{c}_{1}$ is another constant.

Proof. By Theorem 1 it is sufficient to prove for all $\varphi \in C_{0}^{\infty}(\Omega)$

$$
\left|\sum_{\alpha, \beta \in \Gamma^{\prime}} \int_{\Omega} a_{\alpha \beta}(x) D^{\beta} \varphi(x) \overline{D^{\alpha} \varphi(x)} d x\right| \leq\left(c_{0} / 8\right)\|P(D) \varphi\|_{0}^{2}+\underline{c}\|\varphi\|_{0}^{2} .
$$


Indeed, suppose (4.7) to be valid, then by Theorem 1 it follows that

$$
\begin{aligned}
& \operatorname{Re}(L(x, D) \varphi, \varphi)_{0} \geq\left(c_{0} / 4\right)\|P(D) \varphi\|_{0}^{2}-c\|\varphi\|_{0}^{2} \\
&-\left|\sum_{\alpha, \beta \in \Gamma^{\prime}} \int_{\Omega} a_{\alpha \beta}(x) D^{\beta} \varphi(x) \overline{D^{\alpha} \varphi(x)} d x\right| \\
& \geq\left(c_{0} / 8\right)\|P(D) \varphi\|_{0}^{2}-\left(\tilde{c}_{1}+\underline{c}\right)\|\varphi\|_{0}^{2}
\end{aligned}
$$

which is (4.6).

We start with the proof of (4.7). Since $a_{\alpha \beta} \in L^{\infty}(\Omega)$ it follows with some constant $c$ that

$$
\begin{aligned}
& \left|\sum_{\alpha, \beta \in \Gamma^{\prime}} \int_{\Omega} a_{\alpha \beta}(x) D^{\beta} \varphi(x) D^{\alpha} \varphi(x) d x\right| \\
& \quad \leq c\left(\sum_{\substack{\alpha \in \Gamma_{11} \\
\beta \in \Gamma_{12}}}\left\|D^{\beta} \varphi\right\|_{0}\left\|D^{\alpha} \varphi\right\|_{0}\right. \\
& \left.+\sum_{\substack{\alpha \in \Gamma_{21} \\
\beta \in \Gamma_{22}}}\left\|D^{\beta} \varphi\right\|_{0}\left\|D^{\alpha} \varphi\right\|_{0}+\sum_{\alpha, \beta \in \Gamma_{3}}\left\|D^{\beta} \varphi\right\|_{0}\left\|D^{\alpha} \varphi\right\|_{0}\right) .
\end{aligned}
$$

In particular we have for $\alpha \in \Gamma_{11} \cup \Gamma_{22}$ by (4.4)

$$
\begin{aligned}
\left\|D^{\alpha} \varphi\right\|_{0}^{2} & =\int_{\mathbf{R}^{n}} \xi^{2 \alpha}|F \varphi(\xi)|^{2} d \xi \\
& \leq c \int_{\mathbf{R}^{n}}\left(1+|P(\xi)|^{2}\right)|F \varphi(\xi)|^{2} d \xi \\
& \leq c\|P(D) \varphi\|_{0}^{2},
\end{aligned}
$$

where we used for the last estimate Theorem 1.13 in [19]. But for $\alpha \in \Gamma_{12} U$ $\Gamma_{21} \cup \Gamma_{3}$ we find by (4.5) that

$$
\begin{aligned}
\left\|D^{\alpha} \varphi\right\|_{0}^{2} & \leq c \int_{\mathbf{R}^{n}}\left(1+\sum_{l=1}^{n}\left|P^{(l)}(\xi)\right|^{2}\right)|F \varphi(\xi)|^{2} d \xi \\
& \leq c \sum_{l=1}^{n}\left\|P^{(l)}(D) \varphi\right\|_{0}^{2}
\end{aligned}
$$

where again Theorem 1.13 in [19] was used to obtain the last estimate.

Now, using Proposition 2 and the inequality

$$
|a||b| \leq \eta|a|^{2}+\frac{|b|^{2}}{4 \eta}, \quad \eta>0
$$


we get for an arbitrary $\eta>0$

$$
\begin{aligned}
\sum_{\substack{\alpha \in \Gamma_{11} \\
\beta \in \Gamma_{12}}}\left\|D^{\alpha} \varphi\right\|_{0}\left\|D^{\beta} \varphi\right\|_{0} & \leq c\|P(D) \varphi\|_{0}\left(\sum_{l=1}^{n}\left\|P^{(l)}(D) \varphi\right\|_{0}^{2}\right)^{1 / 2} \\
& \leq \eta\|P(D) \varphi\|_{0}^{2}+c(\eta)\|\varphi\|_{0}^{2}, \\
\sum_{\substack{\alpha \in \Gamma_{21} \\
\beta \in \Gamma_{22}}}\left\|D^{\alpha} \varphi\right\|_{0}\left\|D^{\beta} \varphi\right\|_{2} & \leq c\left(\sum_{l=1}^{n}\left\|P^{(l)}(D) \varphi\right\|_{0}^{2}\right)^{1 / 2}\|P(D) \varphi\|_{0} \\
& \leq \eta\|P(D) \varphi\|_{0}^{2}+c(\eta)\|\varphi\|_{0}^{2}
\end{aligned}
$$

and

$$
\begin{aligned}
\sum_{\alpha, \beta \in \Gamma_{3}}\left\|D^{\alpha} \varphi\right\|_{0}\left\|D^{\beta} \varphi\right\|_{0} & \leq c \sum_{l=1}^{n}\left\|P^{(l)}(D) \varphi\right\|_{0}^{2} \\
& \leq \eta\|P(D) \varphi\|_{0}^{2}+c(\eta)\|\varphi\|_{0}^{2} .
\end{aligned}
$$

Taking $\eta$ sufficiently small we finally obtain

$$
\left|\sum_{\alpha, \beta \in \Gamma^{\prime}} \int_{\Omega} a_{\alpha \beta}(x) D^{\beta} \varphi(x) \overline{D^{\alpha} \varphi(x)} d x\right| \leq\left(c_{0} / 8\right)\|P(D) \varphi\|_{0}^{2}+\underline{c}\|\varphi\|_{0}^{2},
$$

which proves the theorem.

\section{The space $H_{0}^{P}(\Omega)$}

Let $P(\xi)$ be a hypoelliptic polynomial. For $\varphi \in C_{0}^{\infty}(\Omega), \Omega \subset \subset \mathbf{R}^{n}$, we set

$$
\|\varphi\|_{P}:=\|P(D) \varphi\|_{0}^{2} \text {. }
$$

By Theorem 1.13 in [19] it follows that on $C_{0}^{\infty}(\Omega)$ a norm is given by (5.1). Again, Plancherel's theorem gives

$$
\|\varphi\|_{P}^{2}=\int_{\mathbf{R}^{\mathbf{n}}}|P(\xi)|^{2}|F \varphi(\xi)|^{2} d \xi
$$

The completion of $C_{0}^{\infty}(\Omega)$ with respect to the norm (5.1) is denoted by $H_{0}^{P}(\Omega)$. By definition $H_{0}^{P}(\Omega)$ is a Hilbert space. By Theorem 11.1.3 in [8], there are constants $d>0, \rho \geq 0$ and $c \geq 0$ such that

$$
\frac{\left|P^{(\alpha)}(\xi)\right|}{|P(\xi)|} \leq c|\xi|^{-|\alpha| d}
$$

holds for all $\xi \in \mathbf{R}^{n},|\xi| \geq \rho$. From the proof of Proposition 2 and Corollary 1.16 in [19] the estimate

$$
\left\|P^{(\alpha)}(D) \varphi\right\|_{0} \leq c\|P(D) \varphi\|_{0}
$$


follows at once for all $\alpha \in \mathbf{N}_{0}^{n}$ and all $\varphi \in C_{0}^{\infty}(\Omega)$. Let $P(\xi)$ be a polynomial of degree $m$ and $\beta \in \mathbb{N}_{0}^{n}$ such that $|\beta|=m$ and $P^{(\beta)}(\xi) \neq 0$. From (5.3) we get with a constant $c^{\prime}$

$$
\left(1+|\xi|^{2}\right)^{m d} \leq c^{\prime}\left(1+|P(\xi)|^{2}\right) .
$$

Finally denote by $H_{0}^{t}(\Omega), t \geq 0$, the usual Sobolev space, that is, the completion of $C_{0}^{\infty}(\Omega)$ with respect to the norm

$$
\|\varphi\|_{t}^{2}=\int_{\mathbf{R}^{n}}\left(1+|\xi|^{2}\right)^{t}|F \varphi(\xi)|^{2} d \xi
$$

We have

THEOREM 3. A. The imbedding of $H_{0}^{P}(\Omega)$ in $H_{0}^{m d}(\Omega)$ is continuous. B. The imbedding of $H_{0}^{P}(\Omega)$ in $L^{2}(\Omega)$ is compact.

PROOF. Since for $t>0$ the imbedding of $H_{0}^{t}(\Omega)$ in $L^{2}(\Omega)$ is always compact (notice that $\Omega$ is a bounded set), we only have to prove part A. But from (5.5) and Theorem 1.13 in [19] we get immediately

$$
\begin{aligned}
\|\varphi\|_{m d}^{2} & =\int_{\mathbf{R}^{n}}\left(1+|\xi|^{2}\right)^{m d}|F \varphi(\xi)|^{2} d \xi \\
& \leq c^{\prime} \int_{\mathbf{R}^{n}}\left(1+|P(\xi)|^{2}\right)|F \varphi(\xi)|^{2} d \xi \\
& \leq c^{\prime \prime}\|P(D) \varphi\|_{0}^{2}=c^{\prime \prime}\|\varphi\|_{P}^{2},
\end{aligned}
$$

which proves part A.

We want to show that the space $H_{0}^{P}(\Omega)$ can be interpreted as a space of functions having generalized homogeneous boundary data. Suppose that $\Omega=$ $Q=\times_{l=1}^{n}\left(a_{l}, b_{l}\right)$ is a cube in $\mathbf{R}^{n}$ with edges parallel to the coordinate axes. The part of $\partial Q$ which is normal to the direction of the unit vector $\varepsilon_{j}$ and which contains no edges is denoted by $\partial_{j} Q$.

THEOREM 4. Let $\alpha \in \mathbf{N}_{0}^{n}, \alpha_{j}>0$ for some $j, 1 \leq j \leq n$. Then for all $x \in \partial_{j} Q$ and all functions $f \in H_{0}^{P}(Q) \cap \bar{C}^{m-1}(Q)$ we have

$$
P^{(\alpha)}(D) f(x)=0 \text {. }
$$

We recall that the degree of $P(\xi)$ is equal to $m$.

PROOF. (see [5], page 28). Let $Z_{h} \subset \bar{Q}$ be a $n$-dimensional cylinder with height $h$, the base of which is an $(n-1)$-dimensional ball in the hyperplane $x_{j}=a_{j}$ with center $x_{0}$ and radius $r_{h}$. We assume that $r_{h}$ is chosen such that the volume of $Z_{h}, \mu\left(Z_{h}\right)$, is equal to $h^{2}$. For $f \in C_{0}^{\infty}(Q)$ we find

$$
P^{(\alpha)}(D) f\left(x_{1}, \ldots, x_{j-1}, a_{j}, x_{j+1}, \ldots, x_{n}\right)=0
$$


for $x_{j}=a_{j}$ and $\sum_{l \neq j} x_{l}^{2}<r_{h}^{2}$. Therefore we have

$$
P^{(\alpha)}(D) f(x)=\int_{a_{j}}^{x_{j}} \frac{\partial}{\partial y_{j}} P^{(\alpha)}(D) f\left(x_{1}, \ldots, x_{j-1}, y_{j}, x_{j+1}, \ldots, x_{n}\right) d y_{j},
$$

and for $x \in Z_{h}$ it follows that

$$
\left|P^{(\alpha)}(D) f(x)\right|^{2} \leq h \int_{0}^{h}\left|\frac{\partial}{\partial y_{j}} P^{(\alpha)}(D) f\left(x_{1}, \ldots, y_{j}, \ldots, x_{n}\right)\right|^{2} d y_{j},
$$

which gives

$$
\int_{Z_{h}}\left|P^{(\alpha)}(D) f(x)\right|^{2} d x \leq h^{2} \int_{Z_{h}}\left|P^{\left(\alpha+\varepsilon_{j}\right)}(D) f(x)\right|^{2} d x,
$$

or

$$
\left(1 / h^{2}\right) \int_{Z_{h}}\left|P^{(\alpha)}(D) f(x)\right|^{2} d x \leq \int_{Z_{h}}\left|P^{\left(\alpha+\varepsilon_{j}\right)}(D) f(x)\right|^{2} d x .
$$

Inequality (5.8) holds for all $f \in C_{0}^{\infty}(Q)$ and therefore by continuity for all $f \in H_{0}^{P}(Q)$. Since $P^{\left(\alpha+\varepsilon_{j}\right)}(D) f \in L^{2}(Q)$ for all $f \in H_{0}^{P}(Q)$ (see (5.4)), it follows that

$$
\int_{Z_{h}}\left|P^{\left(\alpha+\varepsilon_{j}\right)}(D) f(x)\right|^{2} d x \rightarrow 0
$$

as $h \rightarrow 0$. But for $f \in H_{0}^{P}(Q) \cap \bar{C}^{m-1}(Q)$ we have, since $\mu\left(Z_{h}\right)=h^{2}$,

$$
P^{(\alpha)}(D) f\left(x_{0}\right)=\lim _{h \rightarrow 0}\left(1 / h^{2}\right) \int_{Z_{h}}\left|P^{(\alpha)}(D) f(x)\right|^{2} d x
$$

which, together with (5.8) and (5.9), proves the theorem.

\section{A generalized homogeneous Dirichlet problem}

We now pose

PROBLEM 1. Let $\Omega$ be a bounded open set in $\mathbf{R}^{n}, L(x, D)$ a differential operator of form (3.1) and $f \in L^{2}(\Omega)$ a given function. Find all elements $u \in$ $H_{0}^{P}(\Omega)$ such that

$$
B(u, \varphi)=(f, \varphi)_{0}
$$

holds for all $\varphi \in C_{0}^{\infty}(\Omega)$. Here $B(\cdot, \cdot)$ is the sesquilinear form defined by

$$
\begin{aligned}
B(\Psi, \varphi):= & \int_{\Omega} \sum_{i, j=1}^{m} a_{i j}(x) Q_{j}(D) \Psi(x) \overline{P_{i}(D) \varphi(x)} d x \\
& +\int_{\Omega} \sum_{\alpha, \beta \in \Gamma^{\prime}} a_{\alpha \beta}(x) D^{\beta} \Psi(x) \overline{D^{\alpha} \varphi(x)} d x .
\end{aligned}
$$

In order to solve Problem 1 we need the following theorem. 
THEOREM 5. The sesquilinear form $B$ is continuous on $H_{0}^{P}(\Omega)$.

PROOF. It is sufficient to prove all estimate for elements $\Psi, \varphi \in C_{0}^{\infty}(\Omega)$. By definition we have

$$
\begin{aligned}
|B(\Psi, \varphi)| \leq & \left|\int_{\Omega} \sum_{i, j=1}^{m} a_{i j}(x) Q_{j}(D) \Psi(x) \overline{P_{i}(D) \varphi(x)} d x\right| \\
& +\left|\int_{\Omega} \sum_{\alpha, \beta \in \Gamma^{\prime}} a_{\alpha \beta}(x) D^{\beta} \Psi(x) \overline{D^{\alpha} \varphi(x)} d x\right| \\
& \leq M\left(\sum_{i, j=1}^{m}\left\|Q_{j}(D) \Psi\right\|_{0}\left\|P_{i}(D) \varphi\right\|_{0}+\sum_{\alpha, \beta \in \Gamma^{\prime}}\left\|D^{\beta} \Psi\right\|_{0}\left\|D^{\alpha} \varphi\right\|_{0}\right) .
\end{aligned}
$$

Since by our assumptions

$$
\begin{aligned}
& \left\|Q_{j}(D) \varphi\right\|_{0} \leq c_{2}\|P(D) \varphi\|_{0}, \quad 1 \leq j \leq m, \\
& \left\|P_{i}(D) \varphi\right\|_{0} \leq c_{1}\|P(D) \varphi\|_{0}, \quad 1 \leq i \leq m \text {, }
\end{aligned}
$$

and

$$
\left\|D^{\alpha} \varphi\right\|_{0} \leq c\|P(D) \varphi\|_{0}, \quad \alpha \in \Gamma^{\prime},
$$

hold for all $\varphi \in C_{0}^{\infty}(\Omega)$, we get with some constant $c$

$$
|B(\Psi, \varphi)| \leq c\left(\sum_{i, j=1}^{m}\|P(D) \Psi\|_{0}\|P(D) \varphi\|_{0}+\sum_{\alpha, \beta \in \Gamma^{\prime}}\|P(D) \Psi\|_{0}\|P(D) \varphi\|_{0}\right),
$$

which proves the theorem.

Using Theorem 2, Theorem 3.B and Theorem 5 we find by the same arguments as in [2], Theorem 1.14.6

THEOREM 6. Suppose that the differential operator $L(x, D)$ fulfills the conditions C1-C5. Then for Problem 1 Fredholm's alternative holds. That means

The solutions $v \in H_{0}^{P}(\Omega)$ and $w \in H_{0}^{P}(\Omega)$ of the equations

$$
B(v, \varphi)=0 \quad \text { for all } \varphi \in C_{0}^{\infty}(\Omega)
$$

and

$$
B(\varphi, w)=0 \quad \text { for all } \varphi \in C_{0}^{\infty}(\Omega),
$$

respectively, form finite dimensional subspaces $V \subset H_{0}^{P}(\Omega)$ and $W \subset H_{0}^{P}(\Omega)$, respectively. In addition, we have $\operatorname{dim} V=\operatorname{dim} M$. Furthermore, in order that Problem 1 has at least one solution $u \in H_{0}^{P}(\Omega)$, it is necessary and sufficient that the relation

$$
(f, w)_{0}=0
$$

holds for $w \in W$. The solution of Problem 1 is unique up to an element of $V$. 
Notice that we have to use representation theorems for antilinear functionals (see [21], Satz 4.8 and Aufgabe 4.11.e).

\section{Examples and further investigations}

We want to give some examples of differential operators for which the results developed above can be applied. The first example shows that strongly uniformly elliptic operators belong to the class considered here. The second example demonstrates that the same is true for strongly uniformly semielliptic operators. The third example shows that also non-semielliptic operators belong to the class considered here.

EXAMPLE 1. Let

$$
L_{p r}(x, D)=\sum_{|\alpha|=|\beta|=m} D^{\alpha} a_{\alpha \beta}(x) D^{\beta}
$$

and suppose that

$$
\operatorname{Re} L_{\mathrm{pr}}(x, \xi) \geq c|\xi|^{2 m},
$$

and that the coefficients fulfill condition C4. Then the conditions $\mathrm{C1}^{\prime}, \mathrm{C} 2$ and C3 are satisfied. In addition, we can add to $L_{\mathrm{pr}}(x, D)$ any operator

$$
L_{r}(x, D)=\sum_{\substack{|\alpha|,|\beta| \leq m \\|\alpha|+|\beta|<2 m}} D^{\alpha} a_{\alpha \beta}(x) D^{\beta}
$$

with coefficients $a_{\alpha \beta} \in L^{\infty}(\Omega)$ and Theorem 2 will still be valid.

EXAMPLE 2. Suppose that the operator

$$
L_{\mathrm{pr}}(x, D)=\sum_{\substack{\alpha: \tau|=1\\| \beta: \tau \mid=1}} D^{\alpha} a_{\alpha \beta}(x) D^{\beta}
$$

is strongly uniformly semielliptic in the sense of [20], page 327 . Then, under suitable assumptions on the coefficients of (7.3) (see C4), we find that the conditions $\mathrm{C1}^{\prime}, \mathrm{C} 2$ and $\mathrm{C} 3$ are fulfilled.

EXAMPLE 3. Let $Q(\xi)$ be an arbitrary polynomial of degree $m$ with real and constant coefficients and let $k \in \mathbf{N}$ be a given number. Suppose that $R(\xi)$ is a homogeneous, positive definite polynomial of degree $2 k m-2(k-1)$. Furthermore, for $j=1, \ldots, 4$ let $a_{j}: \Omega \rightarrow \mathbb{C}, \Omega \subset \subset \mathbf{R}^{n}$, be functions satisfying $\mathrm{C} 4$ and in addition

$$
\operatorname{Re} a_{j}(x) \geq c_{0}>0, \quad 1 \leq j \leq 4 .
$$

Consider the operator

$$
\begin{aligned}
L(x, D)= & Q(D)^{2 k}\left(a_{1}(x) Q(D)^{2 k}\right)+Q(D)^{2 k}\left(a_{2}(x) R(D)\right) \\
& +R(D)\left(a_{3}(x) Q(D)^{2 k}\right)+R(D)\left(a_{4}(x) R(D)\right) .
\end{aligned}
$$


Then we have

$$
\operatorname{Re} L(x, \xi) \geq c_{0}\left|Q(\xi)^{2 k}+R(\xi)\right|^{2} .
$$

By Theorem 11.1.12 in [8] the polynomial $Q(\xi)^{2 k}+R(\xi)$ is hypoelliptic, but not semielliptic. Hence condition $\mathrm{C} 1$ is satisfied. Conditions C2 and C3 follow from the proof of Theorem 11.1.12 in [8].

In Proposition 5 we prove that if an operator $L(x, D)$ satisfying $\mathrm{C} 1-\mathrm{C} 5$ has continuous coefficients, it is of constant strength in the sense of Definition 13.1.1 in [8] and every operator obtained by freezing the coefficients at some $x_{0}, x_{0} \in \Omega$, is hypoelliptic. In the case where the coefficients are in $\bar{C}^{\infty}(\Omega)$, this gives the following interior regularity result.

THEOREM 7. Let $f \in L^{2}(\Omega) \cap B_{P, k}^{\text {loc }}(\Omega)$ and let $u \in H_{0}^{P}(\Omega)$ be a solution to Problem 1 and suppose that the assumptions of Proposition 5 are fulfilled. Moreover assume that the coefficients of $L(x, D)$ belong to $\bar{C}^{\infty}(\Omega)$. Then $u$ belongs to the space $B_{p, \tilde{L} \mathrm{pr}_{k}^{\mathrm{l}} \mathrm{loc}}^{\mathrm{loc}}(\Omega)$. The space $B_{k, p}^{\mathrm{loc}}(\Omega)$ is defined in [8], page 14, and we have set for some $x_{0} \in \Omega$

$$
L_{\mathrm{pr}}^{0}(\xi)=\left(\sum_{\alpha \in N_{0}^{n}}\left|L_{\mathrm{pr}}^{(\alpha)}\left(x_{0}, \xi\right)\right|^{2}\right)^{1 / 2} .
$$

Proof. By Proposition 5 (see below) we know that the differential operator $L(x, D)$ is of constant strength in $\Omega$ and that for each $x_{0} \in \Omega$ the operator $L\left(x_{0}, D\right)$ is hypoelliptic. Hence, since the coefficients of $L(x, D)$ are assumed to belong to $\bar{C}^{\infty}(\Omega)$, the operator $L(x, D)$ is formally hypoelliptic in the sense of Definition 13.1.1 in [8]. Therefore Theorem 7 follows immediately from Theorem 13.4.1 in [8].

Proposition 5. Let $L(x, D)$ be a differential operator of form (4.1) with continuous coefficients. Suppose in addition that $\mathrm{C} 1, \mathrm{C} 2, \mathrm{C} 3$ and $\mathrm{C} 5$ are fulfilled. Then the operator $L(x, D)$ is of constant strength in $\Omega$ and for each $x_{0} \in \Omega$ the operator $L\left(x_{0}, D\right)$ is hypoelliptic.

Proof. Let $x_{0} \in \Omega$ be an arbitrary point. We prove that $L\left(x_{0}, \xi\right)$ is hypoelliptic. For $\alpha \in \mathbf{N}_{0}^{n}$ we have

$$
L^{(\alpha)}\left(x_{0}, \xi\right)=L_{\mathbf{p r}}^{(\alpha)}\left(x_{0}, \xi\right)+L_{r}^{(\alpha)}\left(x_{0}, \xi\right)
$$


and moreover

$$
\begin{aligned}
\left|L_{\mathrm{pr}}^{(\alpha)}\left(x_{0}, \xi\right)\right| & \leq\left|\partial_{\xi}^{\alpha}\left(\sum_{i, j=1}^{m} P_{i}(\xi) a_{i j}\left(x_{0}\right) Q_{j}(\xi)\right)\right| \\
& =\left|\sum_{i, j=1}^{m} a_{i j}\left(x_{0}\right) \sum_{\beta \leq \alpha}\left(\begin{array}{c}
\alpha \\
\beta
\end{array}\right) \partial_{\xi}^{\alpha-\beta}\left(P_{i}(\xi)\right) \partial_{\xi}^{\beta}\left(Q_{j}(\xi)\right)\right| \\
& \leq c \sum_{i, j=1}^{m} \sum_{\beta \leq \alpha}\left|P_{i}^{(\alpha-\beta)}(\xi) \| Q_{i}^{(\beta)}(\xi)\right| .
\end{aligned}
$$

On the other hand, we have for $\xi \in \mathbf{R}^{n},|\xi| \geq \rho_{0}$,

$$
\left|L_{\mathrm{pr}}\left(x_{0}, \xi\right)\right| \geq \operatorname{Re} L_{\mathrm{pr}}\left(x_{0}, \xi\right) \geq c_{0}|P(\xi)|^{2},
$$

which gives

$$
\frac{\left|L_{\mathrm{pr}}^{(\alpha)}\left(x_{0}, \xi\right)\right|}{\left|L_{\mathrm{pr}}\left(x_{0}, \xi\right)\right|} \leq \frac{c}{c_{0}} \sum_{i, j=1}^{m} \sum_{\beta \leq \alpha} \frac{\left|P_{i}^{(\alpha-\beta)}(\xi)\right|}{|P(\xi)|} \frac{\left|Q_{j}^{(\beta)}(\xi)\right|}{|P(\xi)|} .
$$

But now, by (3.5) it follows that

$$
\lim _{|\xi| \rightarrow \infty} \frac{\left|L_{\mathrm{pr}}^{(\alpha)}\left(x_{0}, \xi\right)\right|}{\left|L_{\mathrm{pr}}\left(x_{0}, \xi\right)\right|}=0
$$

holds for $\alpha \neq 0$, hence $L_{\mathrm{pr}}\left(x_{0}, \xi\right)$ is hypoelliptic. Moreover, we have, using the notation from $\mathrm{C5}$

$$
L_{r}\left(x_{0}, \xi\right)=\sum_{\substack{\alpha \in \Gamma_{11} \\ \beta \in \Gamma_{12}}} a_{\alpha \beta}\left(x_{0}\right) \xi^{\alpha} \xi^{\beta}+\sum_{\substack{\alpha \in \Gamma_{21} \\ \beta \in \Gamma_{22}}} a_{\alpha \beta}\left(x_{0}\right) \xi^{\alpha} \xi^{\beta}+\sum_{\alpha, \beta \in \Gamma_{3}} a_{\alpha \beta}\left(x_{0}\right) \xi^{\alpha} \xi^{\beta} .
$$

By C5 we have

$$
\begin{aligned}
& \left|\sum_{\substack{\alpha \in \Gamma_{11} \\
\beta \in \Gamma_{12}}} a_{\alpha \beta}\left(x_{0}\right) \xi^{\alpha} \xi^{\beta}\right| \leq c|P(\xi)|\left(\sum_{l=1}^{n}\left|P^{(l)}(\xi)\right|^{2}\right)^{1 / 2}, \\
& \left|\sum_{\substack{\alpha \in \Gamma_{21} \\
\beta \in \Gamma_{22}}} a_{\alpha \beta}\left(x_{0}\right) \xi^{\alpha} \xi^{\beta}\right| \leq c\left(\sum_{l=1}^{n}\left|P^{(l)}(\xi)\right|^{2}\right)^{1 / 2}|P(\xi)|
\end{aligned}
$$

and

$$
\left|\sum_{\alpha, \beta \in \Gamma_{3}} a_{\alpha \beta}\left(x_{0}\right) \xi^{\alpha+\beta}\right| \leq c \sum_{l=1}^{n}\left|P^{(l)}(\xi)\right|^{2}
$$


For $|\xi| \rightarrow \infty$ we find using the hypoellipticity of $P(\xi)$, that

$$
\frac{\left|L_{r}\left(x_{0}, \xi\right)\right|}{|P(\xi)|} \leq C \frac{\left.\left.\left|\sum_{l=1}^{n}\right| P^{(l)}(\xi)\right|^{2}\right)^{1 / 2}}{|P(\xi)|}+\frac{\sum_{l=1}^{n}\left|P^{(l)}(\xi)\right|^{2}}{|P(\xi)|^{2}} \rightarrow 0 .
$$

Now Corollary 10.4.8 and Theorem 11.1.9 in [8] imply the hypoellipticity of $L\left(x_{0}, \xi\right)$. We prove that $L(x, \xi)$ is of constant strength in $\Omega$. Since the estimate in (7.8) is independent of $x_{0}$ it is sufficient to show that $L_{\mathrm{pr}}(x, \xi)$ is of constant strength in $\Omega$. But this is proved, if we have show that the polynomials $L_{\mathrm{pr}}\left(x_{0}, \xi\right)$ and $|P(\xi)|^{2}$ are of equal strength for each $x_{0} \in \Omega$. For $\xi \in \mathbf{R}^{n},|\xi|$ sufficiently large, we find that

$$
|P(\xi)|^{2} \leq c \operatorname{Re} L_{\mathrm{pr}}\left(x_{0}, \xi\right) \leq\left|L_{\mathrm{pr}}\left(x_{0}, \xi\right)\right|
$$

hence it follows that

$$
|P(\xi)|^{2} \leq c\left(\sum_{\alpha \in N_{0}^{n}}\left|L_{\mathrm{pr}}^{(\alpha)}\left(x_{0}, \xi\right)\right|^{2}\right)
$$

holds for all $x_{0} \in \Omega$ and $\xi \in \mathbf{R}^{n}$ with a constant $c^{\prime}$ independent of $x_{0}$. On the other hand, we have by (3.3) and (3.4)

$$
\left|L_{\mathrm{pr}}\left(x_{0}, \xi\right)\right| \leq \hat{c}|P(\xi)|^{2} \leq \hat{c} \sum_{\alpha \in N_{0}^{n}}\left|P^{(\alpha)}(\xi)\right|^{2},
$$

again with a constant $\hat{c}$ independent of $x_{0}$. The assertion that the polynomial $|P(\xi)|^{2}$ and $L\left(x_{0}, \xi\right)$ are equally strong follows now from Theorem 10.4.3 in [8].

\section{References}

[1] J. M. Berezanskir, Expansions in eigenfunctions of selfadjoint operators (Translations of Mathematical Monographs 17, Amer. Math. Soc., Providence, Rhode Island, 1968).

[2] A. Friedman, Partial differential equations (Holt, Rinehart and Winston, New York, 1969).

[3] L. Gårding, 'Dirichlet's problem for linear elliptic partial differential equations', Math. Scand. 1 (1953), 55-72.

[4] H.-J. Herrler, 'Entartete lineare elliptische Differentialgleichungen und anisotrope Sobolewräume: Existenz schwacher Lösungen', Z. Anal. Anwendungen 5 (1986), 223-236.

[5] S. Hildebrandt, Lineare elliptische Differentialgleichungen, (Vorlesungsausarbeitung, Mainz, 1966).

[6] L. Hörmander, 'On the regularity of the solutions of boundary problems', Acta Math. 99 (1958), 225-264.

[7] L. Hörmander, The analysis of linear partial differential operators I (Die Grundlehren der mathematischen Wissenschaften 256, Springer Verlag, Berlin-Heidelberg-New YorkTokyo, 1983).

[8] L. Hörmander, The analysis of linear partial differential operators II (Die Grundlehren der mathematischen Wissenschaften 257, Springer Verlag, Berlin-Heidelberg-New YorkTokyo, 1983). 
[9] L. Hörmander, The analysis of linear partial differential operators III (Die Grundlehren der mathematischen Wissenschaften 274, Springer Verlag, Berlin-Heidelberg-New YorkTokyo, 1985).

[10] N. Jacob, 'On generalized Dirichlet problems', Math. Scand. 55 (1984), 245-252.

[11] N. Jacob and B. Schomburg, 'On Gårding's Inequality', Aequationes Math. 31 (1986), 7-17.

[12] B. Malgrange, 'Sur une classe d'opérateurs différentiels hypoelliptiques', Bull. Soc. Math. France 85 (1957), 283-306.

[13] A. Melin, 'Lower bounds for pseudo-differential operators', Ark. Mat. 9 (1971), 117-140.

[14] S. M. Nikol'skily, 'The first boundary problem for a general linear equation', Soviet Math. 3 (1962), 1388-1390.

[15] L. Nirenberg, 'On elliptic partial differential equations', Ann. Scuola Norm. Sup. Pisa (3) 13 (1959), 115-162.

[16] M. Schechter, 'General boundary value problems for elliptic partial differential equations', Comm. Pure Appl. Math. 12 (1959), 457-486.

[17] M. Schechter, 'Remarks on elliptic boundary value problems', Comm. Pure Appl. Math. 12 (1959), 561-578.

(18) M. Schechter, 'Various types of boundary conditions for elliptic equations', Comm. Pure Appl. Math. 13 (1960), 407-425.

[19] M. Schechter, Modern methods in partial differential equations. An introduction (McGrawHill International Book Company, New York, 1977).

[20] F. Stummel, Rand- und Eigenwertaufgabe in Sobolewschen Räumen (Lecture Notes in Mathematics, Springer-Verlag, Berlin-Heidelberg-New York, 1969).

[21] J. Weidmann, Lineare Operatoren in Hilberträumen (Mathematische Leitfäden, Teubner Verlag, 1976).

Mathematisches Institut der Universität

Bismarckstraße 1 1/2

D-8520 Erlangen

Federal Republic of Germany 